•综述・

\title{
植物叶缘形态的发育调控机理
}

\author{
柯锦秀陈多 郭延平 ${ }^{*}$ \\ (生物多样性与生态工程教育部重点实验室, 北京师范大学生命科学学院, 北京 100875)
}

\begin{abstract}
摘要: 生物多样性研究的关键问题之一是表型多样性的形成和演化机制, 因为表型多样性与物种多样性密切相 关, 同时又承载着遗传和环境的变异信息。植物的叶具有丰富的形态多样性, 而叶形多样性很大程度上体现在叶 边缘形态的变异。叶边缘的形态可从全缘、锯齿状到具有不同程度(深浅)和不同式样(羽状或掌状、回数等)的裂 片(在发育研究中复叶的小叶也描述为裂片)。关于叶缘齿/裂的发育调控机制，在拟南芥(Arabidopsis thaliana)、碎 米荠(Cardamine hirsuta)、番茄 (Solanum lycopersicum) 等模式植物中已有较深入的探讨。研究发现, 很多转录因子、 小分子RNA及植物激素对叶齿/裂或小叶的形成具有调控作用, 其中生长素输出途径中的转录因子NAM/CUC、 miR164以及高浓度生长素的反馈调控可能起到核心作用, 而且该调控模块在真双子叶植物中较为保守; TCP类、 SPL类转录因子和其他一些miRNA也在生长素输出途径中发挥作用; 关于KNOX家族转录因子的作用, 虽然多数 研究是围绕复叶的形态建成, 但也有数据显示其在叶裂发育中发挥作用。此外, 对拟南芥和碎米荠等十字花科植 物的研究还发现, 调控基因 $R C O$ 通过抑制小叶裂片之间的细胞增殖而对小叶/叶裂的发育发挥作用。本文综述上述 多角度的研究进展, 并尝试概括叶边缘形态的发育调控网络, 为关于叶缘形态多样性形成机制的研究提供可参考 的切入点。
\end{abstract}

关键词: 叶缘齿/裂; 形态发生; 演化发育; 调控网络

\section{Designing leaf marginal shapes: Regulatory mechanisms of leaf serration or dissection}

Jinxiu Ke, Duo Chen, Yanping Guo*

Ministry of Education Key Laboratory for Biodiversity Science and Ecological Engineering, and College of Life Sciences, Beijing Normal University, Beijing 100875

\begin{abstract}
The mechanism of formation and evolution of phenotypic diversity is one of the key problems in biodiversity science because phenotype diversity is not only a marker of species diversity, but carries the designs adjusted to environments. Plant leaves exhibit a great deal of morphological variation. Such variation is attributed largely to changes of leaf marginal architecture. Leaf marginal shapes can be described as entire, serrate, lobed (varying in depth and patterns) and dissected (also referred to as compound leaf). The molecular mechanism controlling the development of leaf marginal shape has been intensively studied in Arabidopsis thaliana, Cardamine hirsuta, Solanum lycopersicum, and some other plants. Many important regulatory factors such as transcription factors, small RNAs and plant hormones have been found involved in the development of leaf serration or dissection. Among those factors, the transcription factor NAM/CUC, miR164 and auxin in the auxin efflux module play a central role through a feedback loop, and this regulatory module appears to be conserved across the eudicots; the transcription factors TCPs, SPLs and some other miRNAs also take part in the auxin efflux pathway. Transcription factors of the KNOX family play roles in the development of leaf lobes as well although most of the researches about KNOX genes have focused on their regulation of the morphogenesis of compound leaves. In addition, studies in Arabidopsis, Cardamine and other taxa of the Brassicaceae have shown that the gene RCO promotes the development of leaf dissection by repressing growth at the flanks of protrusions generated by CUC-auxin patterning. The present paper reviews the recent progress and integrate the major results of researches about the molecular mechanisms
\end{abstract}


that underlie leaf serration or dissection. We hope this may provide reference for unraveling the morphogenetic origin of the spectacular diversity of leaf marginal shapes.

Key words: leaf serration/dissection; morphogenesis; evolutionary development; regulatory network

生物多样性研究的关键问题之一是表型多样 性的形成和演化机制, 因为表型多样性与物种多样 性密切相关, 同时又承载着环境的变异信息。植物 的叶具有丰富的形态多样性, 一个植物的叶形态不 仅取决于其系统发生和遗传信息, 也有生态因素的 作用, 因为叶片不仅是光合作用的重要场所, 也是 植物感知光、温、水等环境变化的重要器官。对被 子植物而言, 为了进一步适应不同的陆生环境、更 有效地利用光能, 植物在叶形方面发生了巨大的变 化。可以说, 叶的形态变异不仅是植物适应不同环 境的具体体现, 也是植物表型多样性的重要组成部 分。早在 18 世纪, 德国哲学家和诗人歌德(Johann Wolfgang von Goethe)就发现各种叶形的可变异性。 他观察到一个植株上叶的形态转变, 从而推测叶形 的本质在于不断变化(转引自 Chitwood \& Sinha, 2014)。

叶边缘形态是叶形多样性的重要组成部分, 主 要有全缘、锯齿状、不同程度(深浅)和不同式样(羽 状或掌状、回数等)的裂刻等。这些变异具有重要的 生物学意义, 如叶缘裂刻能调节叶面温度和水分流 失, 对干旱、高温等逆境胁迫表现出更强的适应性 (Siso et al, 2001; Kidner \& Umbreen, 2010); 叶缘上 分布的齿或裂刻赋予叶片空间延伸的可塑性, 比全 缘叶更快速响应和竞争有限光源; 此外, 植物学家 早已发现边缘锯齿状的叶能更好地适应低温, 如高 纬度或寒冷地区的植物更多拥有边缘锯齿状或较 深齿裂的叶片(Ledford, 2018), 因此叶齿(serration) / 裂(dissection)的深度可作为地球演化历史中陆地地 块年平均气温的指示物(Wolfe, 1995)。

生物体表型的形成是大量基因表达(包括转录 和翻译)的集合产物(Bonn \& Furlong, 2008), 这些基 因共同构成复杂的调控网络 (gene regulatory network, GRN), 上、下游基因以及相关的小RNA联结 成通路(Carroll, 2008), 通过表达的时空模式和/或 表达水平的改变, 影响着形态特征的发育和建成。

关于叶边缘形态建成的发育调控机制, 在拟南芥 (Arabidopsis thaliana)、碎米荠(Cardamine hirsuta)、烟
草(Nicotiana tabacum)、番茄(Solanum lycopersicum)、 苜宿(Medicago truncatula)、踠豆(Pisum sativum)等模 式植物中已有大量的研究报道(Barkoulas et al, 2007; Blein et al, 2008, 2010; Efroni et al, 2010; Chitwood \& Sinha, 2014; Bar \& Ori, 2015)。叶是茎端分生组织 (shoot apical meristem, SAM)产生的第一类侧生器 官, 其发生和形态建成受茎顶端分生组织生长和分 化的影响。KNOX类基因(Class I KNOTTED1-like homeobox)主要在茎端分生组织中表达, 调控细胞 的分裂和生长, 决定叶原基的形成和复叶形态的发 育, 是迄今为止研究最多的与叶形成和发育密切相 关的调控基因(Hay et al, 2006; Hay \& Tsiantis, 2010; Bar \& Ori, 2015)。叶原基起始后叶向两侧延展, 叶 缘形态随即建成。叶边缘形态多样性也是在复杂的 基因调控网络作用下形成的, 涉及众多的因子, 如 特异基因、转录因子、激素、小RNA等。本文综述 此方面近20年的研究进展, 探讨叶片边缘形态发育 的分子调控机理。

\section{生长素输出(auxin efflux)调控途径}

\section{1 叶边缘锯齿发育的生长素调控模型}

叶片的生长发育可分为 3 个部分重叠的连续过 程, 即: 叶原基形成后的发育起始期、初级形态建 成期(primary morphogenesis, PM) 和次级形态建成 期(secondary morphogenesis, SM)或分化期(differentiation)。在叶片的发育起始期, 叶片细胞主要进 行分裂, 而在后两个时期, 叶片细胞主要进行分化 和生长(Dengler \& Tsukaya, 2001; Bar \& Ori, 2015)。 在叶片的生长发育过程中, 生长素作为最重要的植 物激素之一, 对叶片的形态建成起到了极大的调控 作用。在植物体内, 生长素的运输有极性和非极性 两种方式, 其中极性运输是一种主动运输, 由形态 学上端运至形态学下端, 可以逆浓度梯度而行。这 种极性运输通过相邻的细胞传递, 是由定位在细胞 膜上的输入载体AUX/LAX (AUXIN1/LIKE AUX1) 家族蛋白和输出载体PIN (PIN-FORMED) 家族蛋白 协同作用的结果, 在决定植物形态发育的过程中起 
着非常重要的作用(Benkova et al, 2003; Petrasek et al, 2006; Vandenbussche et al, 2010)。

围绕叶边缘齿或裂刻的生长素调控途径, 有关 生长素输出途径中PIN蛋白及诸多调控因子的研究 较为深入。研究表明, 拟南芥的齿裂叶中, 每个叶 齿突出处都是生长素的高浓度汇聚点, 这与PIN1蛋 白在细胞膜上的极性定位有关。PIN1的动态表达量 数据显示, 叶边缘的PIN1蛋白在表皮细胞中形成一 个极性汇聚点, 生长素的流动方向与PIN1在细胞中 的极性定位方向一致, 从而建立了生长素的最大积 累, 促使此处的叶齿形成。在pin1突变体中, 生长素 的梯度分布被打乱, 沿着整个叶边缘均匀分布, 叶 缘裂刻消失, 形成边缘光滑的全缘叶, 说明依赖于 PIN1的生长素浓度梯度分布决定了叶边缘裂刻的 形成和分布(Benkova et al, 2003; Hay et al, 2006; Barkoulas et al, 2008; Bilsborough et al, 2011)。

PIN1的极性定位受一类转录因子NAM (CUC) 的调控。NAM (NO APICAL MERISTEM) 首先在矮牵 牛(Petunia hybrida)中发现, 其作用主要是决定植物 边缘器官和形态的建成(Souer et al, 1996)。拟南芥 等十字花科植物中的 $N A M$ 直系同源基因有两个, CUC1 和CUC2 (CUP-SHAPED COTYLEDON1，2), 其中 $c u c 2$ 突变体表现为两枚子叶融合为杯状, 相邻 的花萝、雄莣等边缘也相互融合(Aida et al, 1997)。 $N A M$ (CUC1 2)的旁系同源拷贝为 $C U C 3$, 该基因在 功能上与前者存在部分冗余(Vroemen et al, 2003; Hasson et al, 2011)。一系列实验研究表明, CUC/NAM转录因子对叶缘齿/裂的形成起着重要 调控作用: 拟南芥的 $C U C 2$ 和 $C U C 3$ 在叶齿之间凹陷 处均有表达信号, cuc2突变体的叶片裂刻消失变为 全缘, cuc3的叶边缘裂齿程度有所降低; 而 $c u c 1$ 对 叶缘形态基本没有影响。CUC2的作用是在叶片发 育早期决定齿的形成, 而 CUC3则是维持齿的生长 (Nikovics et al, 2006; Kawamura et al, 2010; Bilsborough et al, 2011; Hasson et al, 2011)。另外, 在具 有复叶的植物中, 沉默 NAM/CUC类基因对小叶的 形成也有影响, 其结果使碎米荠和番茄等的小叶融 合、小叶数目和裂齿程度下降(Blein et al, 2008)。

关于 $N A M / C U C$ 类基因对叶边缘形态发育的调 控, 实验数据结合计算机模拟形成了一个基本模型, 即: 叶齿(serration)是一个空间上特异分布的调控 模块的形态学产物, 该模块的核心是 $C U C 2$ 和生长
素的最大作用位点沿发育中的叶边缘间隔分布。具 体的调控模型如图1所示: $C U C 2$ 的表达随叶的生长 在叶边缘呈不连续分布，受其调控，PIN1蛋白在细 胞中重新定位，将生长素以相对的方向逆浓度梯度 运输到一个汇聚点，此处即长出叶齿; 而逐渐积累 的生长素又通过激活一类miRNA- - miR164对 CUC2进行负调控。因此, CUC2和生长素之间形成 一个反馈作用环, CUC2调控生长素的极性汇聚，而 生长素的汇聚又抑制CUC2 的表达，致使CUC2的活 跃区与生长素的最大浓度区相互间隔，映射到表型 上则是：叶齿突出部位是生长素的最大浓度区，而

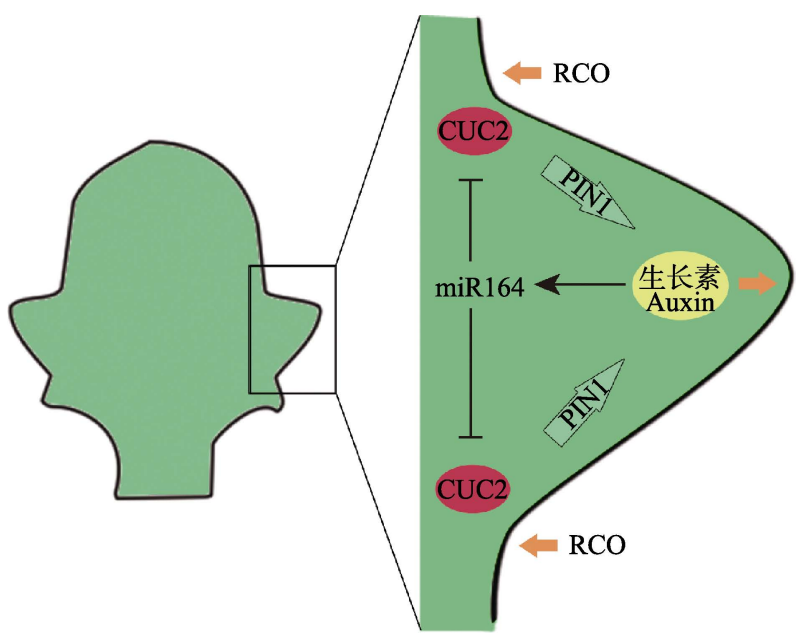

图1 叶缘锯齿发育的调控模型(综合拟南芥和碎米荠中的 研究，基于Barkoulas等(2007)和Runions和Tsiantis (2017)两 文中的图修订而成)。该调控模型的核心是转录因子CUC2 (红色)与生长素(黄色)之间的反馈作用环: CUC2指导PIN1 蛋白在细胞中重新定位, 将生长素从两个方向逆浓度梯度 运到一个汇聚点; 生长素的积累又反过来通过激活 $m i R 164$ 抑制CUC2的表达。CUC2和miR164在共同区域活动，相互 平衡, 调控着生长素的极性运输, 生长素促进叶齿的生长。 另外, 其他生长调节因子也参与修饰叶齿/裂的形态发育, 比如: 碎米荠等十字花科植物的 $R C O$ 类基因抑制叶齿两侧 细胞的生长, 调控更多的齿/裂形态式样。

Fig. 1 A model for the molecular regulation of the development of leaf marginal serration according to studies on Arabidopsis thaliana and Cardamine hirsute (Barkoulas et al, 2007; Runions \& Tsiantis, 2017). At the heart of the model is a feedback loop between CUC2 and auxin activities. CUC2 is required for PIN1-mediated auxin polar transport (horrow arrows); in turn, auxin activity maxima at the tip of the developing serration activates miR164 which represses CUC2 posttranscriptionally and generates an interspersed pattern of auxin maxima and CUC2 expression at the leaf margin. MIR164 and CUC2 are expressed in partially overlapping regions at the sinus of the serrations. Auxin enhances outgrowth of the serrations. There are additional growth regulators modulating leaf growth to shape the form of protrusions, for instance, RCO inhibits growth in indentations, producing more dissected forms. 
凹陷处是 $C U C 2$ 的活跃表达区。在此调控模块中, $C U C 2$ 和 MIR164A 在共同区域表达, 其作用呈动态 平衡; 生长素及其输出载体蛋白PIN1既是叶齿发育 的直接作用者, 又是调控因子间相互作用的介导者 (Nikovics et al, 2006; Barkoulas et al, 2007; Bilsborough et al, 2011; Bar \& Ori, 2014)。

\section{2 真双子叶植物中 NAM/CUC类基因对叶缘形态 调控作用的保守性}

Blein等(2008)比较研究了 5 个真双子叶植物中 $N A M(C U C 1 / 2)$ 和CUC3在叶边缘形态建成中的作用, 发现该类基因具有调控单叶叶齿和复叶上小叶形 成的双重作用, 且这个作用在真双子叶植物中是保 守的。研究表明, 降低番茄植株叶片中 NAM/CUC基 因的表达后, 小叶几乎融合(Berger et al, 2009), 数 目变少, 小叶边缘变为全缘(Blein et al, 2008); 碎米 荠中减少 $N A M / C U C$ 基因的表达后，小叶融合，小 叶和托叶的数目减少(Blein et al, 2008); 在踠豆的 $N A M / C U C$ 基因缺失突变体中检测到这一基因的直 接下游调控基因的表达量明显下降，同时叶形态发 生异常(Blein et al, 2008); 耧斗菜(Aquilegia caeru$l e a$ 的 $N A M$ 基因或 $C U C 3$ 基因被沉默后，小叶叶裂 减少，而同时沉默 $N A M$ 基因和CUC3基因后，小叶 变为全缘(Blein et al, 2008)。此后在苜宿中的研究也 发现, MtNAM 基因缺失突变体的叶片也发生小叶融 合或边缘锯齿减少的现象(Cheng et al, 2012)。

Sha 等 (2018) 首次在菊科植物中检测了 $N A M$-like对于叶齿和羽状分裂的调控作用。菊科萻 属(Achillea)植物的叶缘形态具有丰富的多样性: 齿 叶著 (A. acuminata) 的叶缘为锯齿状; 而亚洲著 (A. asiatica $)$ 的叶则三回羽状全裂, 裂片成细丝状。Sha 等(2018)克隆出两种植物中的 NAM-like基因— AacNAM和AasNAM, 发现它们的表达时空模式有 显著差异, 即: 在齿叶著中, AacNAM仅在叶边缘齿 的相应位置表达, 随着幼叶的生长其表达量持续下 降; 而亚洲著中, AasNAM在幼叶边缘以及伸长的裂 片边缘(对应二回裂片的位置)均有表达信号, 且表 达量随着幼叶的生长上下波动。进一步的分析发现 两个物种的 $N A M$-like在启动子区域存在一个较大 片段(1210碱基)的缺失-插入变异, 这可能意味着顺 式调控元件的差异。该研究不仅支持了 $N A M$ (CUC1/2) 在真双子叶植物中叶缘形态调控作用的 保守性, 也表明单回叶裂和多回叶裂的差异很可能
与NAM-like的时空表达模式差异相关，即：多回裂 片的发生可能是调控模块周期性运作的结果。

综上所述，在多个植物类群中均检测到 $C U C / N A M$ 基因功能的缺失会导致叶边缘齿裂程度 的降低, 说明 CUC/NAM对叶边缘形态发育的调控 作用在真双子叶植物中是保守的。

\section{3 通过生长素输出途径影响叶缘形态发育的其 他因子}

植物 miRNA负调控内源性靶基因，通过与靶 基因转录物的部分或完全结合来识别和裂解这些 转录物。在叶发育过程中, miRNAs与其靶向基因共 同作用于生长素的输出通路，从而调控叶缘齿/裂 的发育。研究表明, 生长素最大积累瞬时诱导 MIR164的表达，miR164通过对靶基因CUC2的转录 后负调控来影响器官原基的边界建立。利用T-DNA 插入方法获得的拟南芥mir164突变体, MIR164表达 量降低导致CUC2表达量升高，叶片裂刻加深(Nikovics et al, 2006), 而当miR164过表达时，CUC2表 达降低, 导致拟南芥叶变全缘、碎米荠小叶变少 (Rubio-Somoza et al, 2014)。CUC2的旁系同源基因 CUC3 缺乏 $m i R 164$ 的结合位点而不受其调控, 但 CUC3 与 CUC2之间存在相互作用, CUC2 能促进 CUC3活性，两者形成的异源二聚体在叶片发育过 程中调节叶齿/裂的发育和叶缘复杂性的建成(Chitwood \& Sinha, 2014; Rubio-Somoza et al, 2014)。

另一组能调控叶缘复杂性的转录因子是受 miR319负调控的 TCPs (TEOSINTE BRANCHED 1/CYCLOIDEA/PCF) 家族成员。MIR319A 和 MIR319B单突变一定程度上抑制拟南芥叶缘齿裂的 形成，双突变使得拟南芥叶变全缘(Koyama et al, 2017)。过表达MIR319, TCP水平降低，导致拟南芥 叶齿增多、碎米荠小叶增多(Palatnik et al, 2003; Daniel et al, 2004)。Rubio-Somoza和他的团队将 miR164-CUC 与 miR319-TCP两条 miRNA 调节通路 在蛋白水平联系起来, 表明TCP4能分别与CUC2和 CUC3形成二聚体, 从而阻止CUC2-CUC3二聚体的 形成，导致叶齿/裂程度降低。其他研究者的相关研 究也都表明, TCP类转录因子可负调控CUC类基因 而发挥对叶边缘形态的调控作用(Palatnik et al, 2003; Daniel et al, 2004; Chitwood \& Sinha, 2014; Rubio-Somoza et al, 2014; Koyama et al, 2017)。

此外, miR156在植物营养生长过程中发挥着关 
键性作用, 且与叶缘形态发育紧密相关。拟南芥中, miR156的活性在植物叶发育过程中呈逐渐下降趋 势; 通过操纵 $m i R 156$ 的活性能减慢或加快叶复杂性 的获得(Rubio-Somoza et al, 2014)。miR156的作用是 通过其靶向转录因子家族的 SPLs (SQUAMOSA PROMOTER BINDING PROTEIN-LIKE)而实现的。 研究发现, SPL9能与 TCP4形成二聚物, 因此减少 TCP 与 CUC的结合, 间接调控叶边缘形态的复杂 性。Rubio-Somoza等(2014)根据实验结果解释了叶 发育过程中叶缘的变化: 叶发育早期幼嫩时, miR156水平较高，抑制了SPL9的活性使之无法与 TCPs结合, 从而更多的TCPs得以与CUCs结合, 阻 止了CUC2-CUC3二聚体的形成，也就阻碍了叶齿、 小叶的形成; 随着叶龄增长, miR156水平下降, 导 致SPL9水平上升, 大量与TCPs发生反应形成二聚 体，从而抑制了后者与CUC2或CUC3的结合，更多 的CUC2-CUC3二聚体促进了叶齿或小叶数目的增 多。基于这一研究结果, Chitwood和Sinha (2014)解 释了植株个体发育过程中叶异型性(heteroblasty)的 miR156-SPL9调控原理。

上述调控模块中的 $\mathrm{TCP}$ 转录因子的活性又受到 TIE1 (TCP INTERACTOR-CONTAINING EAR MOTIF PROTEIN1)转录抑制因子的作用: 在叶缘形态 发育中 TIE1 能招募共抑制因子 TOPLESS (TPL)/TOPLESS-RELATED (TPR)在蛋白水平上抑 制TCP的活性，因此，过表达TIE1能引起叶边缘裂 刻的加剧 (Tao et al，2013); 而 TIE1 又与 TEAR1 (TIE1-ASSOCIATED RING-TYPE E3 LIGASE1)有 相互作用, TEAR1及其同源基因发生突变可引起叶 片边缘锯齿增多(Zhang et al, 2017)。秦跟基课题组 (Tao et al, 2013; Zhang et al, 2017)根据其实验结果 提出一种新的精确调控TCP活性的叶缘形态发育调 控机制：TIE1招募TPL/TPRs共同抑制TCP的活性， 而TEAR1通过介导TIE1的降解来解除TIE1对TCP 的抑制，从而正调控TCP的活性。可见, TIE1和 TEAR1通过对TCP的调控而间接影响了叶缘的形 态发育。

\section{生长素输入(auxin influx)途径的调控因于}

生长素输入载体蛋白 AUX1/LAX家族成员对 于稳定由PIN输出载体蛋白主导的生长素极性运输 具有重要作用。研究发现, AUX1/LAX和PIN家族的
基因在顶端分生组织中都有表达，叶原基的形成主 要依赖PIN的活性，但也有 AUX1的作用(Kramer, 2004; Heisler \& Jonsson, 2006)。AUX1/LAX在叶原基 中也有表达，对叶缘的形态发育有一定的作用(Bilsborough et al, 2011)。AUX1/LAX1/LAX2三种AUX1/ $L A X$ 家族基因在拟南芥早期叶发育过程中有明显的 特异表达模式( LAX1在叶尖端表达并随着叶的生长 在叶缘齿裂的位置表达、 $L A X 2$ 随叶生长逐渐局限于 叶脉部位表达、 $A U X 1$ 在分生组织表面以及沿着新 长出的叶原基边缘表达)，当AUX1/LAX1/LAX2 同时 突变时, 叶边缘锯齿会减小, 但双突变或单突变对 叶缘均无明显影响(Kasprzewska et al, 2015)。

3 生长素途径中与叶缘形态相关的其他唒自 控因子

植物中一种名为 EPFL2 (EPIDERMAL PATTERNING FACTOR-LIKE)的肽和ERECTA (ER)家 族成员也参与叶边缘锯齿的形态发生过程(Tameshige et al, 2016)。当拟南芥中的EPFL2或ER发生突 变时, 叶片边缘会变得圆滑, 并检测到生长素在整 个叶缘区分布(在具有齿的野生型叶片中，叶边缘 的生长素只在齿的位置累积)。EPFL2与ERECTA形 成配体一受体对，从而抑制生长素在叶齿处的响应， 而生长素又反过来抑制EPFL2的表达，形成负反馈 环。该反馈系统在叶缘生长过程中维持着生长素的 响应式样(Tameshige et al, 2016)。

另外, 目前的研究表明, JAGGED (JAG)、 JAGGED LATERAL ORGANS (JLO)、Trifoliate (Tf) 等也能通过生长素途径发挥叶边缘形态发育的调 控作用; 同时它们还能影响KNOX调控通路, 这将 在下面的段落讨论。

\section{KNOX家族基因调控途径}

\subsection{KNOX家族基因对叶缘形态发育的调控作用}

KNOX 属于 KNOTTED1-LIKE HOMEOBOX $(K N O X)$ 基因家族(Vollbreeht et al, 1991), 在拟南芥 中有 4 个成员: SHOOT MERISTEMLESS (STM), KNAT1 (又称BREVIPEDICELLUS, BP), KNAT2以及 KNAT6 (Hake et al, 2004)。番茄中的KN2 (TKN2)和 $T K N 1$ 以及玉米中的 KNOTTED1 (KN1)也为 $K N O X$ 家 族的成员(Hay \& Tsiantis, 2010)。

$K N O X$ 家族成员在植物顶端分生组织中表达, 
其调控作用对叶和花的发生、发育都是必不可少且 是保守的(Bharathan et al, 2002; Piazza et al, 2010)。 $K N O X$ 基因在叶原基形成的起始时期表达下调或没 有表达, 但随后在叶片发育过程中其表达存在物种 特异性, 这与叶缘形态的多样性有关(Piazza et al, 2010)。在绝大多数双子叶植物中, KNOX的表达活 性与叶缘的复杂性高度相关, 在很多具羽状复叶或 叶缘有裂刻的物种中可检测到 KNOX在叶片中表达 (Bharathan et al, 2002; Piazza et al, 2010), 如在碎米 荠、番茄的复叶里, $K N O X$ 基因在叶片发育过程中有 表达。拟南芥中存在全缘叶的植株类型, Piazza等 (2010)在用15个基因位点的DNA序列信息对拟南芥 属及十字花科其他支系的物种进行系统发生分析 基础上, 推断拟南芥中的齿裂叶为祖征, 全缘叶为 衍生，而齿裂的消失与叶片中STM (KNOX家族成员) 的表达缺失有关, 因为在其他叶缘分裂的物种的发 育叶片中均检测到 STM的表达, 且敲减其表达后叶 裂消失; 进一步的分析显示该表达缺失可能与STM 启动子区域序列的变异有关(Piazza et al, 2010)。另 外, 在叶片中异常表达 $K N O X$ 基因可引起叶缘形态 的强烈反应：过表达 $K N O X$ 基因时，拟南芥、烟草以 及番茄的 $L a$ 突变体(为单叶)出现增多的叶裂; 降低 碎米荠中STM的表达时, 小叶发育被严重抑制, 甚 至形成单叶, 而过表达 $K N O X$ 则引起小叶增加(Hay \& Tsiantis, 2006; Shani et al, 2009; Efroni et al, 2010); 在野生番茄叶中过表达玉米的KN1, 产生超级复叶, 小叶数目剧增(Hareven et al, 1996; Efroni et al, 2010)。可见, 无论是小叶还是叶缘裂刻的发育都受 到 $K N O X$ 家族基因的调控, 其作用机制可能是 $K N O X$ 通过负调控赤霉素的生物合成基因或促进细 胞分裂素的生物合成而改变激素之间的平衡, 从而 控制叶缘形态的发育(Efroni et al, 2010; Moon \& Hake, 2011)。然而也有研究表明, 具多裂叶的豆类 植物叶片上并没有 $K N O X$ 基因的表达(Piazza et al, 2010)。

\section{2 通过KNOX途径影响叶缘形态发育的其他因子}

$K N O X$ 的时空表达受多个不同调控途径因子的 影响, 很多因子并不直接影响叶缘形态的发育, 但 由于其能直接或间接促进或抑制 $K N O X$ 基因, 因此 也导致了叶缘形态的变化。惠麦霞等(2012)对于这 些调控因子的作用已作了详细综述, 本文仅作概述 和补充。
$K N O X$ 类基因的表达受 $A R P$ 基因(名称源于拟南 芥 的 ASYMMETRIC LEAVES1 (AS1), 玉 米的 ROUGH SHEATH2 (RS2)和金鱼草的PHANTASTICA) 的抑制。这类基因编码MYB转录因子，在形成侧生 器官的细胞中表达并抑制KNOX的活性。拟南芥中, 突变体 $a s 1$ 的叶缘产生裂刻，叶原基中 $K N O X$ 类基因 (BP，KNAT2，KNAT6)表达普遍上调(Byrne et al, 2002)。AS1的这个作用机制是它与AS2形成二聚体 复合物直接结合到KNOX的启动子区域，共同抑制 $K N O X$ 基因在器官原基中的表达(Byrne, 2005; Moon \& Hake, 2011; Bar \& Ori, 2015)。

BLADE ON PETIOLE1 (BOP1)和BOP2属于 BTB蛋白家族, 两者能形成二聚物, 发挥转录激活 因子的功能。 $B O P 1$ 和 $B O P 2$ 在侧生器官基部表达, 在叶基部表达的 $B O P$ 能直接正调控 $A S 2$ ，抑制该区 域KNOX基因的表达(Jun et al, 2010)。bop1和bop2双 突体的叶片变大, 在叶柄处长出叶齿、叶翅等(Ha et al, 2003, 2010; Norberg et al, 2005)。因此, BOP通过 抑制叶基部和叶柄处 $K N O X$ 的表达来保证正常的叶 片形态建成(Ha et al, 2010; Wang et al, 2015)。

JAGGED LATERAL ORGANS (JLO) 被认为是拟 南芥中器官边界建立的“调节器” (Wang et al, 2015), 属于LATERAL ORGAN BOUNDARY DOMAIN (LBD) 基因家族成员, 在分生组织和器官原基的边界特异 表达。JLO能诱导叶片中 KNOX家族STM和KNAT1 的表达，并抑制PIN的基因表达，当 $J L O$ 在拟南芥叶 片异位表达时可产生深裂、叶柄减短的叶片(Borghi et al, 2007)。

$J A G G E D(J A G)$ 编码具有 $\mathrm{C} 2 \mathrm{H} 2$ 锌指结构的转录 因子。 $J A G$ 功能缺失能引起拟南芥的叶产生叶裂; 番茄中的JAG同源基因是 LYRATE (LYR), lyr突变株 产生更多小叶，而 $L Y R$ 的过表达则引起小叶融合。 可见, $J A G / L Y R$ 可能通过与 $K N O X$ 以及生长素途径中 的因子相互作用, 共同参与叶裂深度和小叶数目的 调控(Dinneny et al, 2004; David-Schwartz et al, 2009; Bar \& Ori, 2014)。

SAWTOOTH属于BEL1-LIKE HOMEODOMAIN (BLH)蛋白家族成员。SAWTOOTH1 (BLH2/SAW1) 和SAWTOOTH2 (BLH4/SAW2)在侧生器官中表达, 可负调控 $K N O X$ 家族基因 $B P$ 的表达。saw1或saw 2 的 单突变不引起明显的表型变化, 但 $s a w 1$ 和saw2双突 变体可使拟南芥叶片齿裂加深，叶边缘外卷。在as1 
突变植株中过表达SAW1, 可抑制as1对叶表型的影 响和 $B P$ 在叶片上的表达。可见, BLH2/SAW1 和 $B L H 4 / S A W 2$ 通过抑制一个或多个 $K N O X$ 基因的表达 参与叶缘形态的建成(Kumar et al, 2007)。

另外, Trifoliate $(T f)$ 编码一个在拟南芥中与 LOF1和LOF2蛋白相关的MYB-like转录因子, 它在 叶缘和叶腋表达。在番茄植株的个体发育过程中, 早期叶原基中 $T f$ 表达水平增加, 促进了叶缘形态的 复杂性; $t f$ 突变体的叶片明显简单化, 叶齿和叶裂减 少。研究表明, $\mathrm{Tf}$ 能影响叶基部原基中 KNOX的活性, 并且, 植物生长素需要 $\mathrm{Tf}$ 的活性才能启动小叶形 成。所以Tf可能通过调控 KNOX以及生长素活性来 影响叶缘形态发育(Naz et al, 2013)。

\section{其他因子的调控途径}

REDUCED COMPLEXITY (RCO) 起源于一个祖 先基因的重复事件, 即LMI1 (LATE MERISTEM IDENTITY 1)-like基因在十字花科的系统发育早期 (Aethionema属分化之后)发生的一次基因复制事 件。此次重复产生了 LMI1-type和RCO-type两个拷贝, 在碎米荠和琴叶拟南芥(Arabidopsis lyrata) 等植物 中, 存在两个 $R C O$-type基因(源于 $R C O$-type的再次 重复), 但是拟南芥(A. thaliana)的基因组中却不存
在RCO-type的序列(Vlad et al, 2014)。在碎米荠中 $R C O$ 仅活跃在生长的叶片中, 对于叶缘形状发育有 重要的影响。研究人员在比较研究了碎米荠的全裂 叶与拟南芥的浅齿叶后发现，碎米荠复杂的叶片形 状可归因于 $R C O$-type基因: 当 $R C O$ 基因的功能被敲 除时, 碎米荠的叶不再产生小裂片; 而将 $R C O$ 转入 拟南芥后，其近圆形单叶则发育为深裂叶。形态上， 只有单个祖先拷贝LMI1-like的Aethionema arabicum 与拟南芥一样也具有单叶, 因此研究人员根据系统 发生分析及基因的功能分析推测, 具有复叶的碎米 荠等是因为拥有发生了新功能化的RCO-type基因, 而RCO-type在拟南芥的演化过程中丢失，导致拟南 芥在形态上发生了返祖现象, 其叶片变成边缘具浅 状齿的单叶(Vlad et al, 2014)。另外，比较荠属中具 不同叶裂水平的物种发现, $R C O$ 的旁系同源基因也 可能参与了十字花科植物叶形多样性的形成(Bar \& Ori, 2014)。

$R C O$ 调控叶裂的机制在于能确保小叶/裂片形 成位置之间的叶边缘区域细胞的增殖和生长受到 阻遏，其功能仅限于对叶缘部分区域的生长起抑制 作用。在碎米荠中, $R C O$ 基因缺失并不会产生除叶 形变化以外的任何其他可见变化(Vlad et al, 2014)。 另外，在十字花科中， $R C O$ 并没有对生长素的运输

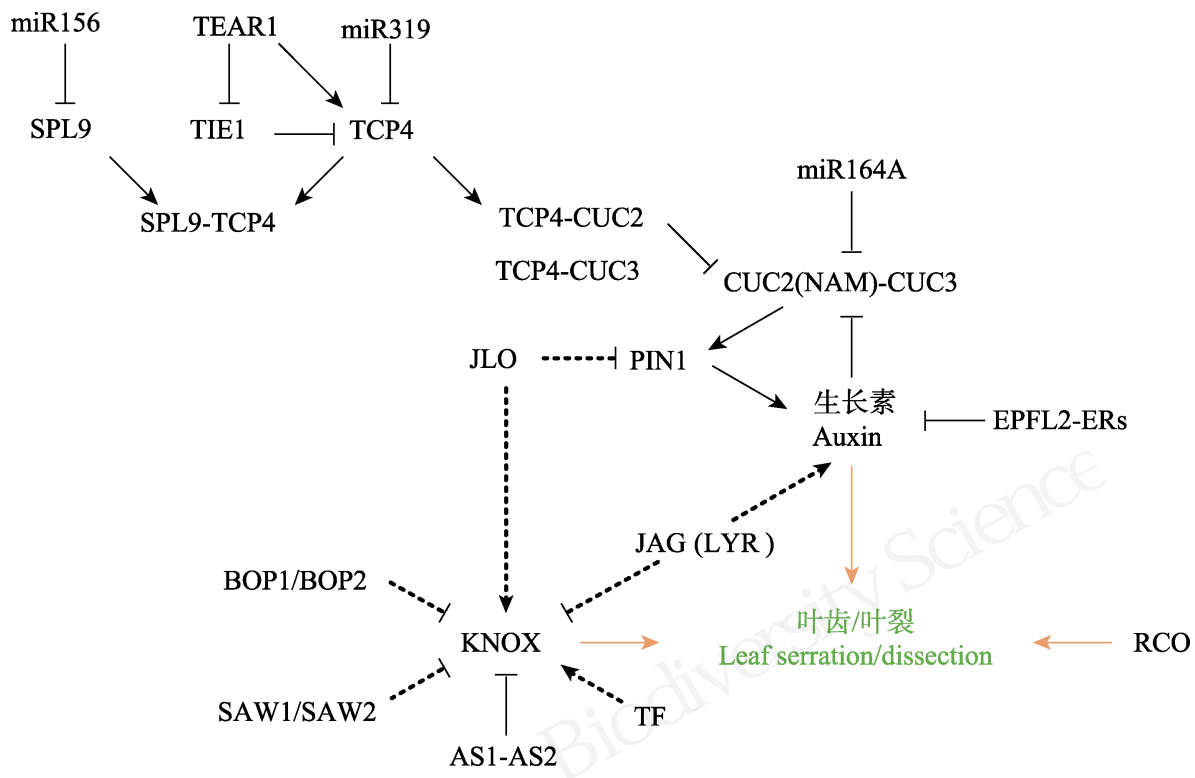

图2 叶边缘形态发育调控网络图, 示各因子间的相互作用。实线表示已有大量研究清晰阐明的相互作用; 虚线则表示相关研 究报道较少，尚不太明确的相互作用; 橙色箭头表示促进叶齿/裂表型的形成。

Fig. 2 The molecular regulating network underlining the development leaf serration/dissection. The solid lines indicate the relatively clear interactions between molecules, while the dotted lines suggest unsure interactions reported by a few studies. The orange arrows suggest promotion of leaf serration/dissection. 
产生影响(Vlad et al, 2014), 但它能在CUC-auxin调 控模块的基础上抑制叶齿两侧细胞的生长, 从而改 变齿裂的深度, 产生更多的齿裂形态式样(Runions \& Tsiantis, 2017) (图1)。

综合上述叶边缘形态发育调控方面的研究进 展, 概括其调控网络如图2所示。

\section{6 展望}

植物叶的发育是一个非常有趣的过程。首先, 叶发育的过程具有很强的可塑性，不同的环境决定 着不同的叶片大小和形态; 而另一方面，植物叶片 的发育又遵循着一个基本模式，是一个比较保守的 有序过程。叶边缘发育是叶形态建成的最后步骤 (Tang et al, 2016), 很大程度上决定了植物叶形的复 杂性。

近年来的研究成果揭示了叶缘的形态发育受 多种调控因素的复杂调控, 如转录因子、小分子 RNA及植物激素等, 其中围绕生长素输出途径中的 一些重要因子, 如CUC、TCP、SPL以及miRNAs等 的研究最多，也最为深入和成熟，这些因子之间的 相互作用关系也越来越清晰。但从进化的角度来看, 推动叶边缘或叶形变异的因素是什么? 又是什么 因素使得叶形的改变能长时间保持并在一个种群 中被固定下来? 利用RNA-seq, Ostria等(2016)发现 光照能调节NAC家族基因的表达水平从而调节叶 边缘复杂性。目前的研究表明, 作用于TCP活性的 miR156/SPL 通路对光照强度很敏感, 从而影响 CUC 与 TCP通路之间的相互反应 (Chitwood \& Sinha, 2016) (见前述), 这从另一个角度说明了这几 个因子在叶边缘形态方面重要的调控作用。

随着研究的深入, 越来越多的调控因子被发现 与叶边缘发育相关, 但它们在复杂调控网络中的作 用机制仍不清楚。要阐明叶缘形态的多样性与其复 杂易变的调控网络之间的联系, 还有很多难题有待 解决。

从进化和系统发育的角度看, 植物类群形态的 多样性主要源于相关的发育调控因子的作用或网 络的演变。然而, 关于叶发育调控机制和网络的演 化, 人们的认识还十分有限, 尚需以大量非模式植 物为材料进行比较发育生物学研究。此外, 为了解 影响叶缘形态变异的环境因子, 需要综合生态、遗 传发育和进化生物学的研究方法, 即所谓
Eco-Evo-Devo, 探求自然居群和同质种植条件下叶 形的变异。随着分子生物学技术和生物信息学方法 的发展，人们有机会获取越来越多的信息，期待着 Eco-Evo-Devo这个新的综合不断揭开生物表型多 样性形成和演化的神秘面纱。

\section{参考文献}

Aida M, Ishida T, Fukaki H, Fujisawa H, Tasaka M (1997) Genes involved in organ separation in Arabidopsis: An analysis of the cup-shaped cotyledon mutant. The Plant Cell, 9, 841-857.

Bar M, Ori N (2014) Leaf development and morphogenesis. Development, 141, 4219-4230.

Bar M, Ori N (2015) Compound leaf development in model plant species. Current Opinion in Plant Biology, 23, 61-69.

Barkoulas M, Galinha C, Grigg SP, Tsiantis M (2007) From genes to shape: Regulatory interactions in leaf development. Current Opinion in Plant Biology, 10, 660-666.

Barkoulas M, Hay A, Kougioumoutzi E, Tsiantis M (2008) A developmental framework for dissected leaf formation in the Arabidopsis relative Cardamine hirsuta. Nature Genetics, 40, 1136-1141.

Benkova E, Michniewicz M, Sauer M, Teichmann T, Seifertova D, Jurgens G, Friml J (2003) Local, efflux-dependent auxin gradients as a common module for plant organ formation. Cell, 115, 591-602.

Berger Y, Harpaz-Saad S, Brand A, Melnik H, Sirding N, Alvarez JP, Zinder M, Samach A, Eshed Y, Ori N (2009) The NAC-domain transcription factor GOBLET specifies leaflet boundaries in compound tomato leaves. Development, 136, 823-832.

Bharathan G, Goliber TE, Moore C, Kessler S, Pham T, Sinha NR (2002) Homologies in leaf form inferred from KNOX gene expression during development. Science, 296, 1858-1860.

Bilsborough GD, Runions A, Barkoulas M, Jenkins HW, Hasson A, Galinha C, Laufs P, Hay A, Prusinkiewicz P, Tsiantis M (2011) Model for the regulation of Arabidopsis thaliana leaf margin development. Proceedings of the National Academy of Sciences, USA, 108, 3424-3429.

Blein T, Hasson A, Laufs P (2010) Leaf development: What it needs to be complex. Current Opinion in Plant Biology, 13, 75-82.

Blein T, Pulido A, Vialette-Guiraud A, Nikovics K, Morin H, Hay A, Johansen IE, Tsiantis M, Laufs P (2008) A conserved molecular framework for compound leaf development. Science, 322, 1835-1839.

Bonn S, Furlong E (2008) cis-Regulatory networks during development: A view of Drosophila. Current Opinion in Genetics and Development, 18, 513-520.

Borghi L, Bureau M, Simon R (2007) Arabidopsis JAGGED 
LATERAL ORGANS is expressed in boundaries and coordinates KNOX and PIN activity. The Plant Cell, 19, 17951808.

Byrne ME (2005) Shoot development-genetic interactions in the meristem. Biochemical Society Transactions, 33, 1499-1501.

Byrne ME, Simorowski J, Martienssen RA (2002) ASYMMETRIC LEAVES1 reveals KNOX gene redundancy in Arabidpsis. Development, 129, 1957-1965.

Carroll S (2008) Evo-devo and an expanding evolutionary synthesis: A genetic theory of morphological evolution. Cell, 134, 25-36.

Cheng X, Peng J, Ma J, Tang Y, Chen R, Mysore KS, Wen J (2012) NO APICAL MERISTEM (MtNAM) regulates floral organ identityand lateral organ separation in Medicago truncatula. New Phytologist, 195, 71-84.

Chitwood DH, Sinha NR (2014) Plant development: Small RNAs and the metamorphosis of leaves. Current Biology, 24, R1087-R1089.

Chitwood DH, Sinha NR (2016) Evolutionary and environmental forces sculpting leaf development. Current Biology, 26, R297-R306.

Chitwood DH, Sinha NR (2004) Small RNAs and the metamorphosis of leaves. Current Biology, 22, 1087-1089.

David-Schwartz R, Koenig D, Sinha NR (2009) LYRATE is a key regulator of leaflet initiation and lamina outgrowth in tomato. The Plant Cell, 21, 3093-3104.

Dengler NG, Tsukaya H (2001) Leaf morphogenesis in dicotyledons: Current issues. International Journal of Plant Sciences, 162, 459-464.

Dinneny JR, Yadegari R, Fiseher RL, Yanofsky MF, Weigel D (2004) The role of JAGGED in shaping lateral organs. Development, 131, 1101-1110.

Efroni I, Eshed Y, Lifschitz E (2010) Morphogenesis of simple and compound leaves: A critical review. The Plant Cell, 22, 1019-1032.

Ha CM, Jun JH, Fleteher JC (2010) Control of Arabidopsis leaf morphogenesis through regulation of the YABBY and KNOX families of transcription factors. Genetics, 186, 197-206.

Ha CM, Kim GT, Kim BC, Jun JH, Soh MS, Ueno Y, Maehida Y, Tsukaya H, Nam HG (2003) The BLADE-ON-PETIOLE 1 gene controls leaf pattern formation through the modulation of meristematic activity in Arabidopsis. Development, 130, 161-172.

Hake S, Smith HMS, Holtan H, Magnani E, Mele G, Ramirez J (2004) The role of KNOX genes in plant development. Annual Review of Cell Developmental Biology, 20, 125-151.

Hareven D, Gutfinger T, Parnis A, Eshed Y, Lifschitz E (1996) The making of a compound leaf: Genetic manipulation of leaf architecture in tomato. Cell, 84, 735-744.

Hasson A, Plessis A, Blein T, Adroher B, Grigg S, Tsiantis M, Boudaoud A, Damerval C, Laufs P (2011) Evolution and diverse roles of the CUP-SHAPEDCOTYLEDON genes in Arabidopsis leaf development. The Plant Cell, 23, 54-68.

Hay A, Tsiantis M (2006) The genetic basis for differences in leaf form between Arabidopsis thaliana and its wild relative Cardamine hirsuta. Nature Genetics, 38, 942-947.

Hay A, Barkoulas M, Tsiantis M (2006) ASYMMETRIC LEAVES1 and auxin activities converge to repress BREVIPEDICELLUS expression and promote leaf development in Arabidopsis. Development, 133, 3955-3961.

Hay A, Tsiantis M (2010) KNOX genes: Versatile regulators of plant development and diversity. Development, 137, 3153-3165.

Heisler MG, Jonsson H (2006) Modeling auxin transport and plant development. Journal of Plant Growth Regulation, 25, 302-312.

Hui MX, Zhang LG, Zhang MK (2012) Research progress on the molecular mechanism of leaf margins. In: Proceedings of the 14th Symposium of the Crucifers Subdivision of the Chinese Society for Horticultural Science, pp. 52-59. (in Chinese) [惠麦霞, 张鲁刚, 张明科 (2012) 植物叶缘发育 分子机理研究进展. 见: 中国园艺学会十字花科分会第十 四届学术研讨会论文集, 52-59页.]

Jun JH, Ha CM, Fletcher JC (2010) BLADE-ON-PETIOLE1 coordinates organ determinacy and axial polarity in Arabidopsis by directly activating ASYMMETRIC LEAVES2. Plant Cell, 22, 62-76.

Kasprzewska A, Carter R, Swarup R, Bennett M, Monk N, Hobbs JK, Fleming A (2015) Auxin influx importers modulate serration along the leaf margin. The Plant Journal, 83, 705-718.

Kawamura E, Horiguchi G, Tsukaya H (2010) Mechanisms of leaf tooth formation in Arabidopsis. The Plant Journal, 62, 429-441.

Kidner CA, Umbreen SU (2010) Why is leaf shape so variable. International Joumal of Plant Developmental Biology, 4 (Special Issue 1), 64-75.

Koyama T, Sato F, Ohme-Takagi M (2017) Roles of miR319 and TCP transcription factors in leaf development. Plant Physiology, 175, 874-885.

Kramer EM (2004) PIN and AUX/LAX proteins: Their role in auxin accumulation. Trends in Plant Science, 9, 578-582.

Kumar R, Kushalappa K, Godt D, Pidkowich MS, Pastorelli S, HePworth SR, Haughn GW (2007) The Arabidopsis BEL1-LIKE HOMEODOMAIN proteins SAW1 and SAW2 act redundantly to regulate KNOX expression spatially in leaf margins. The Plant Cell, 19, 2719-2735.

Ledford H (2018) The lost art of looking at plants. Nature, 553, 396-398.

Moon J, Hake S (2011) How a leaf gets its shape. Current Opinion in Plant Biology, 14, 24-30.

Naz AA, Raman S, Martinez CC, Sinha NR, Schmitz G, Theres K (2013) Trifoliate encodes an MYB transcription factor that modulates leaf and shoot architecture in tomato. Proceedings 
of the National Academy of Sciences, USA, 110, 24012406.

Nikovics K, Blein T, Peaucelle A, Ishida T, Morin H, Aida M, Laufs P (2006) The balance between the MIR164A and CUC2 genes controls leaf margin serration in Arabidopsis. The Plant Cell, 18, 2929-2945.

Norberg M, Holmlund M, Nilsson O (2005) The BLADE ON PETIOLE genes act redundantly to control the growth and development of lateral organs. Development, 132, 22032213.

Ostria GE, Ranjan A, Zumstein K, Chitwood DH, Kumar R, Townsley BT, Ichihashi Y, Corcuera LJ, Sinha NR (2016) Transcriptomic analysis suggests a key role for SQUAMOSA PROMOTER BINDING PROTEIN LIKE, NAC and YUCCA genes in the heteroblastic development of the temperate rainforest tree Gevuina avellana (Proteaceae). New Phytologist, 210, 694-708.

Palatnik JF, Allen E, Wu X, Schommer C, Schwab R, Carrington JC, Weigel D (2003) Control of leaf morphogenesis by microRNAs. Nature, 425, 257-263.

Petrasek J, Mravec J, Bouchard R, Blakeslee JJ, Abas M, Seifertova D, Niewska JW, Tadele Z, Kubes M, Covanova M, Dhonukshe P, Skupa P, Benkova E, Perry L, Krecek P, Lee OR, Fink GR, Geisler M, Murphy AS, Luschnig C, Zazimalova E, Frim J (2006) PIN proteins perform a rate-limiting function in cellular auxin efflux. Science, 312, 914-918.

Piazza P, Bailey CD, Cartolano M, Krieger J, Cao J, Ossowski S, Sehneeberger K, He F, de Meaux J, Hall N, Maeleod N, Filatov D, Hay A, Tsiantis M (2010) Arabidopsis thaliana leaf form evolved via loss of KNOX expression in leaves in association with a selective sweep. Current Biology, 20, 2223-2228.

Rubio-Somoza I, Zhou CM, Confraria A, Martinho C, von Born P, Baena-Gonzalez E, Wang JW, Weigel D (2014) Temporal control of leaf complexity by miRNA-regulated licensing of protein complexes. Current Biology, 24, 2714-2719.

Runions A, Tsiantis M (2017) The shape of things to come: From typology to predictive models for leaf diversity. American Journal of Botany, 104, 1437-1441.

Sha S, Chen D, Liu M, Li KL, Jiang CK, Wang DH, Guo YP (2018) To be serrate or pinnate: Diverse leaf forms of yarrows (Achillea) are linked to differential expression patterns of NAM genes. Annals of Botany, 121, 255-266

Shani E, Burko Y, Ben-Yaakov L, Berger Y, Amsellem Z, Goldshmidt A, Sharon E, Ori N (2009) Stage-specific regulation of Solanum lycopersicum leaf maturation by Class KNOTTED1-LIKE HOMEOBOX proteins. The Plant Cell, 21, 3078-3092.

Siso S, Camarero JJ, Gil-Pelegrin E (2001) Relationship between hydraulic resistance and leaf morphology in broad leaf Quercus species: A new interpretation of leaf lobation. Trees, 15, 341-345.

Souer E, van Houwelingen A, Kloos D, Mol J, Koeset R (1996) The No Apical Meristem gene of petunia is required for pattern formation in embryos and flowers and is expressed at meristem and primordia boundaries. Cell, 85, 159-170.

Tameshige T, Okamoto S, Lee JS, Aida M, Tasaka M, Torii KU, Uchida N (2016) A secreted peptide and its receptors shape the auxin response pattern and leaf margin morphogenesis. Current Biology, 26, 2478-2485.

Tang Y, Zhao CY, Tan ST, Xue HW (2016) Arabidopsis type II Phosphatidylinositol 4-Kinase PI4K $\gamma 5$ regulates auxin biosynthesis and leaf margin development through interacting with membrane-bound transcription factor ANAC078. PLoS Genetics, 12, e1006252.

Tao Q, Guo D, Wei B, Zhang F, Pang C, Jiang H, Zhang J, Wei T, Gu H, Qu LJ, Qin G (2013) The TIE1 transcriptional repressor links TCP transcription factors with TOPLESS/ TOPLESS-RELATED corepressors and modulates leaf development in Arabidopsis. The Plant Cell, 25, 421-437.

Vandenbussche F, Petrasek J, Zadnikova P, Hoyerova K, Pesek B, Raz V, Swarup R, Bennett M, Zazimalova E, Benkova E, Van Der Straeten D (2010) The auxin influx carriers AUX1 and LAX3 are involved in auxin-ethylene interactions during apical hook development in Arabidopsis thaliana seedlings. Development, 137, 597-606.

Vlad D, Kierzkowski D, Rast MI, Vuolo F, Ioio RD, Galinha C, Gan X, Hajheidari M, Hay A, Smith RS, Huijser P, Bailey CD, Tsiantis M (2014) Leaf shape evolution through duplication, regulatory diversification, and loss of a homeobox gene. Science, 343, 780-783.

Vollbreeht E, Veit B, Sinha N, Hake S (1991) The developmental gene Knotted-1 is a member of a maize homeobox gene family. Nature, 350, 241-243.

Vroemen CW, Mordhorst AP, Albrecht C, Kwaaitaal MACJ, de Vries SC (2003) The CUP-SHAPED COTYLEDON3 gene is required for boundary and shoot meristem formation in Arabidopsis. The Plant Cell, 15, 1563-1577.

Wolfe JA (1995) Paleoclimatic estimates from tertiary leaf assemblages. Annual Review of Earth Planetary Sciences, 23, 119-142.

Wang Q, Hasson A, Rossmann S, Theres K (2015) Divide et impera: Boundaries shape the plant body and initiate new meristems. New Phytologist, 209, 485-498.

Zhang J, Wei B, Yuan R, Wang J, Ding M, Chen Z, Yu H, Qin G (2017) The Arabidopsis RING-type E3 ligase TEAR1 controls leaf development by targeting the TIE1 transcriptional repressor for degradation. The Plant Cell, 29, 243-259. 